\title{
Notice of Corrections
}

Re: Bolourani S, Tayebi MA, Diao L, Wang P, Patel V, Manetta F, Lee PC. Using machine learning to predict early readmission following esophagectomy. J Thorac Cardiovasc Surg. May 29, 2020 [Epub ahead of print].

The authors discovered an error in the code used in the experimentation of the 3 methods presented in Figure 4 and, by extension, the Central Figure. The error is in the section where 4-fold cross validation of models are performed and introduces a "leak" between the training set and testing set. This error exists for all 3 models presented. The implication of this error is that the predictive ability of all 3 models are overestimated. After correcting the error, the area under the receiver operating characteristic of the models diminishes by between approximately $1 \%$ to $20 \%$, depending on the model. The performance metrics mentioned in the article, and the blended metrics presented in Table E7, are also overestimated. However, the discussion regarding Figure 4 remains the same: the random forest model was the most accurate model among those presented, and the sensitivity of the model increases after applying the NearMiss method, which comes at the cost of decreasing specificity and accuracy, both models have far superior predictive ability than logistic regression, and the order of blended metrics presented in Table E7 remains intact. This error does not impact the methodology, the rest of the results, the discussion, and the conclusion of this article.

The authors would like to convey their sincere apologies to the readers and editors for the inconvenience this has caused. The errors have been corrected in the online version of the article before final publication.

Check for updates

Re: Pawale A, McCarthy PM. Commentary: Residual mitral regurgitation: The fork in the road. J Thorac Cardiovasc Surg. 2020;160:1193-4.

The Editors wish to inform readers that Amit Pawale, MD, is a co-author of the Commentary with Patrick McCarthy, MD, and Dr Pawale is also a co-author of the paper reviewed by the Commentary: El-Eshmawi AA, Boateng AC, Pawale P, Pandis A, Bhatt D, Sun H, et al. Second crossclamp to perfect degenerative mitral valve repair: decision-making algorithm, safety, and outcomes. J Thorac Cardiovasc Surg. 2020;160:1181-90. 